\title{
A NOTE ON THE DEFINITION OF ARC-SETS ${ }^{1}$
}

\author{
W. L. AYRES
}

The interesting subsets of a Peano space which were later called the arc-sets or $A$-sets were defined independently and in a different manner by G. T. Whyburn ${ }^{2}$ and the author, ${ }^{3}$ and each studied the properties of these sets which have proved so useful in the later theory. The similarity of the properties led shortly to the conjecture that a relation existed between the two definitions, and investigation proved that they were equivalent. These are the properties (1) and (3) of the theorem of the present note. Two more equivalent properties, the properties (2) and (4) of the present note, were observed immediately. ${ }^{4}$

The present note introduces two new properties and shows that all six are equivalent, so that any one may be taken as the definition of the arc-sets or $A$-sets.

Theorem. For a nondegenerate ${ }^{5}$ subset $A$ of a Peano space $P$ the following six properties are equivalent:

(1) There is a set $H$ such that $A$ is the set of points of all arcs of $P$ whose end points belong to $H$.

(2) A contains every arc of $P$ whose end points belong to $A$.

(3) $A$ is a connected collection of cyclic elements of $P$.

(4) For any connected subset $C$ of $P$, the set $A \cdot C$ is connected.

(5) Every set separating 6 two points of $A$ in $A$ separates them in $P$.

(6) $A$ is connected, $\bar{A} \cdot \bar{C}$ is a single point for each component $C$ of $P-A$, and this point $y$ belongs to $A$ if $\bar{A}$ contains two continua $S$ and $T$ such that $S \cdot T=y$.

1 Presented to the Society, December 1, 1939.

2 Concerning the structure of a continuous curve, American Journal of Mathematics, vol. 50 (1928), pp. 167-194.

${ }^{3}$ Concerning the arc-curves and basic sets of a continuous curve, Transactions of this Society, vol. 30 (1928), pp. 567-578.

${ }^{4}$ Properties (1), (3), and (4) were proved equivalent by W. L. Ayres, Concerning the arc-curves and basic sets of a continuous curve, second paper, Transactions of this Society, vol. 31 (1929), pp. 595-612. That (1), (2), (3), and (4) are equivalent was remarked with an indication of proof by C. Kuratowski and G. T. Whyburn, Sur les éléments cycliques et leurs applications, Fundamenta Mathematicae, vol. 16 (1930), pp. 305-331. See page 321.

${ }^{5}$ Even in the trivial degenerate case, (1), (2), (4), (5), and (6) are still equivalent if we define a point $x$ as being an arc from $x$ to $x$.

${ }^{6}$ We use the word separating here in the weak or coupure sense, that is, a set $K$ separates two points $x$ and $y$ in a set $A$ if $x+y \subset A$ and there exists no connected set $C$ such that $x+y \subset C \subset A-K$. 
$(1) \rightarrow(2)$. This has been proved by the author. ${ }^{7}$

$(2) \rightarrow(3)$. It follows directly from (2) that $A$ is connected, in fact, arc-wise connected. Let $E$ be a cyclic element of $P$ such that $A \cdot E \neq 0$. If $A \cdot E$ is a single point, this point is a cut point of $P$ and thus is itself a cyclic element. Suppose $A \cdot E \supset x+z$ and let $y$ be any other point of $E$. Then $E$ contains an $\operatorname{arc}^{8} x y z$ which belongs to $A$ by property (2). Hence $E$ belongs to $A$.

$(3) \rightarrow(4)$. This has been proved by Kuratowski and Whyburn. ${ }^{9}$

$(4) \rightarrow(5)$. Let $K$ denote a set separating $x$ and $y$ in $A$. If there exists a connected set $C$ such that $x+y \subset C \subset P-K$, then $C \cdot A$ is a connected subset of $A-K$ containing $x+y$ by (4). Then $K$ does not separate $x$ and $y$ in $A$.

$(5) \rightarrow(6)$. The set $A$ is connected, for otherwise the null set would separate two points of $A$ in $A$, which it could not in $P$.

If $C=y$ is a degenerate component of $P-A, \bar{C} \cdot \bar{A}=y$. As $A$ is connected, any degenerate component of $P-A$ is a non-cut point of $P$. Then $y$ is an end point or belongs to some simple closed curve $J$ of $P$. As $y$ is a point component of $P-A, J$ contains two points $x$ and $z$ of $A$. Let $\alpha$ and $\beta$ denote the subarcs $x y$ and $y z$ of the arc $x y z$ of $J$. There exists an open set $U$ such that $U \supset \alpha-y$ and $\bar{U} \cdot \beta=y$. This open set may be obtained by summing all open spheres whose centers are points $w$ of $\alpha-y$ and whose radii are less than the distance from $w$ to $\beta$. Then $K=F(U)-y$ separates $x$ and $z$ in $A$ but does not separate them in $P$ as it contains no point of the arc $x y z$. Hence every degenerate component of $P-A$ is an end point. As $y$ is an end point of $P$, there do not exist in $P$, and thus not in $\bar{A}$, two continua $S$ and $T$ such that $S \cdot T=y$.

Suppose that $C$ is nondegenerate and $\bar{A} \cdot \bar{C} \supset x+z$. As $C$ is connected, $C-x-z \neq 0$. We shall show that $P$ contains an arc $x y z$, where $y \subset C$. If there is a point $y$ in $P$ which separates $x$ and $z$, then the connectivity of $C$ gives $C \supset y$ and any arc $x z$ of $P$ contains $y$. If no point of $P$ separates $x$ and $z$, there is a true cyclic element $E \supset x+z$. As each component of $P-E$ is an open set and has just one limit point in $E$, it follows that $P-E+x+z$ contains no connected subset containing $x+z$. But as $C+x+z$ is such a connected set, $C$ must contain a point $y$ of $E$ distinct from $x$ and from $z$. Then $E$ contains ${ }^{8}$ an arc $x y z$.

As $x+z \subset \bar{A} \cdot \bar{C}$, each neighborhood of $x$ and $z$ respectively contains points $x^{\prime}$ and $z^{\prime}$ of $A$. If these neighborhoods are chosen sufficiently

${ }^{7}$ Arc-curves, Theorem 3.

${ }^{8}$ W. L. Ayres, Continuous curves which are cyclicly connected, Bulletin de l'Académie Polonaise des Sciences et des Lettres, 1928, pp. 127-142, Theorem 3.

${ }^{\bullet}$ Loc. cit., Theorem 8.5. 
small, there exist small arcs $x x^{\prime}$ and $z z^{\prime}$ such that the set $x x^{\prime}+x y z+z z^{\prime}$ contains an arc $x^{\prime} y z^{\prime}$. Then just as above we may construct an open set $U$ such that $F(U)-y$ separates $x^{\prime}$ and $z^{\prime}$ in $A$ but not in $P$. Thus $\bar{A} \cdot \bar{C}$ is a single point for every component $C$ of $P-A$.

Suppose continua $S$ and $T$ exist such that $S+T \subset \bar{A}$ and $S \cdot T=y$. Suppose $y \notin A$. Then $S$ contains a point $x$ of $A$, for otherwise $S$ belongs to a component $C_{1}$ of $P-A$ and $\bar{A} \cdot \bar{C}_{1} \supset S$, which is not a single point. Similarly $T$ contains a point $z$ of $A$. By the same process as outlined above, there exists an open set $U$ such that $U \supset S-y$, $\bar{U} \cdot T=y$. Then $F(U)-y$ separates $x$ and $z$ in $A$ but not in $P$ as it contains no point of the connected $S+T$.

$(6) \rightarrow(1)$. Let $H=A$. Then the set of points of all arcs of $P$ whose end points belong to $H$ certainly contains $A$. Suppose there is an arc $x y z$ such that $x+z \subset H=A$ and $y \$ A$. Let $C$ denote the component of $P-A$ containing $y$. The subarc $x y$ contains a point $x$ of $A$ and a point $y$ of $C$, and thus contains a point of $\bar{A} \cdot \bar{C}$. Similarly the subarc $y z$ contains a point of $\bar{A} \cdot \bar{C}$. But $\bar{A} \cdot \bar{C}$ is a single point. Hence $\bar{A} \cdot \bar{C}=y$.

As $y$ was any interior point of the arc which belonged to $P-A$ and we showed that $\bar{A} \supset y$, we have $\bar{A} \supset$ arc $x y z$. Let $S=$ subarc $x y$ and $T=$ subarc $y z$. We have $S+T \subset \bar{A}, S \cdot T=y$, and hence $y \subset A$ by property (6).

The University of Michigan 